\title{
Research on two - stage Exponential Acceleration Algorithm for Stepping Motor
}

\author{
Tianjie Xia ${ }^{a}$, Ying $\mathrm{Li}^{\mathrm{b}}$ \\ School of Mechanical \& Power Engineer, East China University of Science and Technology, \\ Shanghai 200237, China \\ aq516403882@163.com, byingli163@sina.com
}

\begin{abstract}
Keywords: Stepping Motor, PLC, Critical Load Curve, Two-Stage Exponential Acceleration, Iterative Algorithm.
\end{abstract}

\begin{abstract}
In order to solve the problem that the stepping motor in the manipulator is frequently stopped and the conventional index algorithm can not make full use of the output torque under the high speed heavy load,the two-stage exponential acceleration algorithm for stepper motor is proposed.Based on the critical load curve, the two-stage exponential type and the conventional exponential acceleration scheme are analyzed and compared, which proves that the former has better fastness. The critical load curve fitting is carried out by Matlab software, and the best point are selected by w-t equation. The two-stage exponential acceleration iterative algorithm is designed to reduce the dispersion of the speed-increasing process and improve the smoothness of the stepping motor acceleration process.Finally, the experimental results show that the motor speed curve under the two-stage exponential control is in accordance with the theoretical curve, and the feasibility of the method is proved.
\end{abstract}

\section{Introduction}

As the industry automation level of our country becoming more advanced, to alleviate people's working intensity and improve production proficiency, it is more common to use mechanical arm. Because of the stability and robustness of the combination of PLC and stepper motor, it is now using in the control of mechanical arm [1].

Stepper motor a open-loop control motor that is transforming electrical pulse signals into angular displacement or linear displacement. It is the main component of modern digital program control system and it is now widely used in various automatic control systems [2]. The advantage of this control process is simple and the open-loop accuracy is high. But because of the torque frequency characteristic, the stepper motor will have the phenomenon that the output torque will decrease with the increase of frequency in actual operation.

In order to solve the motor failure caused by torque frequency characteristics and the optimization of motor acceleration and deceleration. There are roughly three kinds of control methods [3]: 1) Linear acceleration and deceleration curves. This method can not round up the acceleration speed, uniform speed and deceleration speed well. It is mainly applicable to the occasions where the speed of the control system is slow and the process of acceleration speed is not high; 2) Exponential acceleration and deceleration curve. The method has good rapidity and high motion accuracy; 3) S acceleration and deceleration curve. This method has the advantages of smooth control and smooth transition at all stages, but the segmentation calculation is more complicated.In order to enable the mechanical arm to start quickly and smoothly to achieve the target speed, the acceleration algorithm is usually used to complete the acceleration process. Exponential algorithm has good rapidity and is suitable for mechanical arm with fast changing speed requirement. 


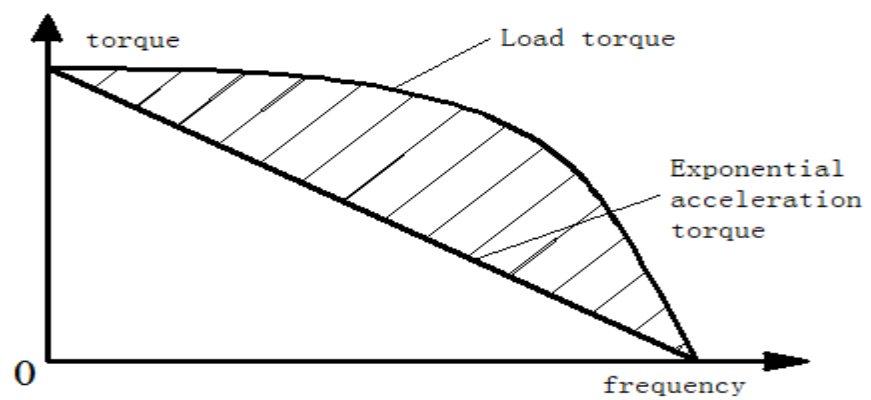

Figure 1. Stepping Motor Output Load Torque - Frequency Curve

However, according to the existing literature [4], pointing out that the exponential curve acceleration process can not take full advantage of the motor output torque, such as the diagonal section in Figure 1 of the acceleration process and the surplus torque. In order to maximize the load torque and make the stepper motor have more acceleration at each moment, a two stage exponential acceleration scheme based on the moment frequency characteristic curve is proposed.

\section{Design of Two - stage Exponential Acceleration Scheme}

The stepper motor works as a controller to give a pulse, the motor rotates a step angle, by changing the frequency of pulse transmission to change the motor rotation speed. When the motor rotates, the electrical inductance of the winding will form a reverse electromotive force; the higher the frequency, the greater the reverse EMF, in its role, increase the motor with frequency and phase current decreases, resulting in decreased torque $\mathrm{Td}$ [5].

The dynamic characteristics of a motor can be described by its kinetic model [6] (two order differential equations). :

$J \frac{d^{2} \theta}{d t^{2}}+\beta \frac{d \theta}{d t}+k \theta+T_{z}=T_{d}$

(1) formula: $\mathrm{J}$ - total rotation inertia of the system (including the inertia of the step motor no-load and the moment of inertia of the load translation under load)

$\theta--$ rotor angle

B-- damping (including mechanical damping and electromagnetic damping) coefficients

$\mathrm{K}$ - proportionality factor with theta as a function

$\mathrm{Tz}$ - the friction resistance moment and the sum of other resistance moments associated with theta

$\mathrm{Td}$ - electromagnetic drive torque generated by stepping motor

Ignore the load item with the scaling factor of the position as a function:

$\mathrm{Ja}+\beta \omega+T_{z}=T_{d}$

Among them, a ( $\mathrm{rad} / \mathrm{s} 2)$ is angular acceleration, and ( $\mathrm{rad} / \mathrm{s})$ is angular velocity.

According to (2) formula, it can be found that the higher the motor speed, the smaller the acceleration value can be obtained at this moment. In order to avoid the actual torque shortage, we should avoid the critical state of torque balance.

$$
J a<T_{\mathrm{d}}-T_{z}-\beta \omega
$$

(3) in $\mathrm{Td}$, the torque frequency characteristic curve decreases with the increase of frequency $\mathrm{f}$, and $\mathrm{Tz}$ is the intrinsic resistance moment of the system. $\omega=\theta \mathrm{f}, \theta$ is the step angle of stepping motor, so the linear frequency increases linearly with the increase of frequency.

Figure 2 shows the torque of the system changes in the stepper motor system as the frequency increases. The solid line is the motor output torque $\mathrm{Td}$, coarse dashed line is the output torque minus system resistance torque (Td-Tz), thin lines for critical load curve (Td-Tz- $\beta \omega$ ), the range is surrounded by diagonal acceleration torque can reach Ja. In order for the motor to achieve the target speed more quickly, then the value of Ja should be close to the fine solid line as far as possible to obtain the maximum angular acceleration at each moment. 

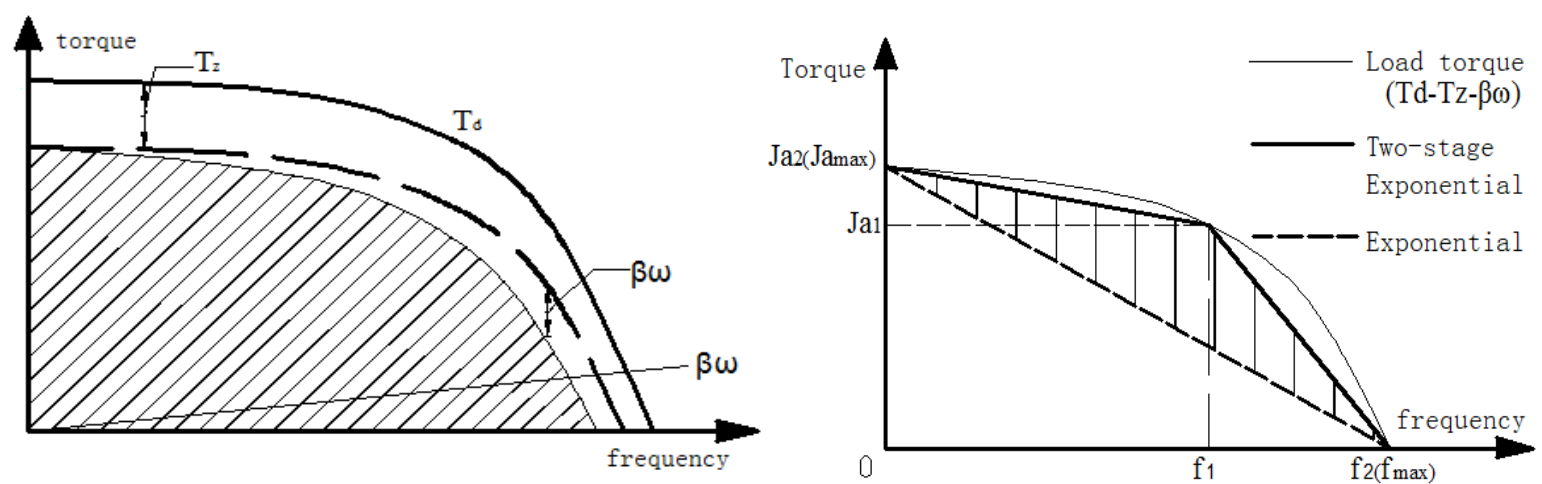

Figure 2. The Torque Distribution Figure 3.Two - Stage Exponential Acceleration Curve

As shown in Figure 3, based on the critical load curve, two exponential accelerated moment frequency relation lines are constructed. Compared to the traditional exponential acceleration scheme, the two stage exponential scheme can utilize the motor output torque more frequently at each acceleration step.

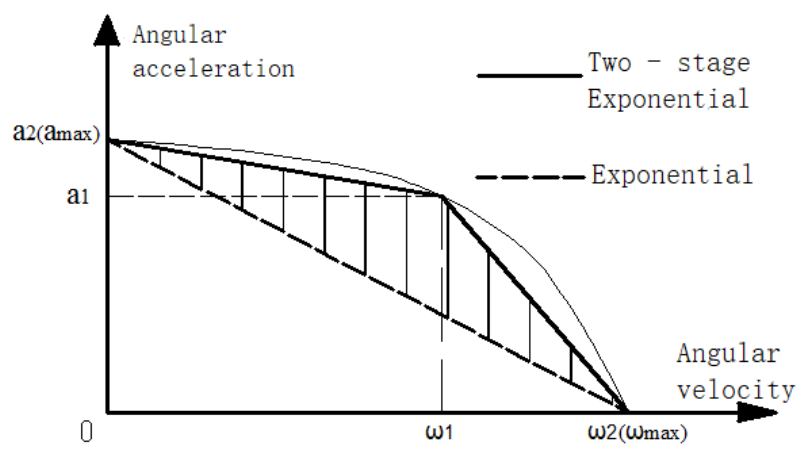

Figure 4. a- $\omega$ Diagram

Because angular speed $\omega$ has linear relationship with frequency $f, \omega=\theta f(\theta$ is step angle), and a is proportional to Ja, so there is figure 4. By Figure 4, two exponential and exponential acceleration compared to a- $\omega$ method, can be found in $0-\omega_{2}$, the former has the angular acceleration greater than the latter, so theoretically proved that this method is rapid acceleration of higher.

As can be seen from Figure 4, the two exponential accelerated algorithm is divided into two segments with a break point $(\mathrm{a} 1, \omega 1)$. The first segment starts with the initial $\omega=\omega 0$, and the $\mathrm{a}=\mathrm{a} 2$ accelerates until $\omega=\omega 1$, and the acceleration time is T1; the second segment continues to accelerate to the target angular velocity $\omega 2$ at initial $\omega=\omega 1$ and $\mathrm{a}=\mathrm{a} 1$.

$$
\begin{aligned}
& a=\frac{d \omega}{d \mathrm{t}}= \begin{cases}\frac{a_{1}-a_{2}}{\omega_{1}} \omega+a_{2} & 0-\omega_{1} \\
\frac{a_{1}}{\omega_{1}-\omega_{2}}\left(\omega-\omega_{2}\right) & \omega_{1}-\omega_{2}\end{cases} \\
& \omega= \begin{cases}\left(\frac{a_{2} \omega_{1}}{a_{1}-a_{2}}+\omega_{0}\right) e^{\frac{a_{1}-a_{2}}{\omega_{1}} t}-\frac{a_{2} \omega_{1}}{a_{1}-a_{2}} \\
\left(\omega_{1}-\omega_{2}\right) e^{\frac{a_{1}}{\omega_{1}-\omega_{2}} t t_{1}}+\omega_{2}\end{cases}
\end{aligned}
$$

For the $\mathrm{T}$ integral, the two segments are substituted into the boundary values $\mathrm{t}=0$, omega $=$ Omega $0, \mathrm{t}=\mathrm{t} 1$, omega $=$ Omega 1 , and two exponential type Omega $-\mathrm{t}$ equations can be obtained.

(5) is the two accelerating process, the $\omega$-t equation. Because of the exponential feature, the acceleration at the end of the second phase is infinitely close to the target velocity, $\omega 2$, so that the acceleration time becomes infinitely long. So you can set the acceleration target to $0.99 \omega 2$, and then hit the value to a small jump to $\omega_{2}$.

According to the (5) formula, the total time of two runs to $0.99 \omega_{2}$ is obtained: 


$$
t=\frac{\omega_{1} \ln \frac{a_{1} \omega_{1}}{a_{2} \omega_{1}+\omega_{0}\left(a_{1}-a_{2}\right)}}{a_{1}-a_{2}}+\frac{\omega_{2}-\omega_{1}}{a_{1}} \ln \left(\frac{100\left(\omega_{2}-\omega_{1}\right)}{\omega_{2}}\right)
$$

According to (6), it is easy to find that in the two exponential accelerated scheme, the selection of the break point $(\mathrm{A} 1, \omega 1)$ will affect the total time of the two stage acceleration. Because the critical load curve is fitted to the data given by the motor manufacturer and the system parameters, it is not easy to obtain its function form. The approximate curve $\mathrm{ax}=\mathrm{f}(\omega \mathrm{x})$ can be obtained by the curve fitting toolbox of Matlab software, and the substitution formula (6) is available:

$$
t_{\omega x}=\frac{\omega_{x}}{f_{\left(\omega_{x}\right)}-a_{2}} \ln \frac{f_{\left(\omega_{x}\right)} \omega_{x}}{a_{2} \omega_{x}+\omega_{0}\left(f_{\left(\omega_{x}\right)}-a_{2}\right)}+\frac{\omega_{2}-\omega_{x}}{f_{\left(\omega_{x}\right)}} \ln \left(\frac{100\left(\omega_{2}-\omega_{x}\right)}{\omega_{2}}\right)
$$

By importing formula (7) into Matlab software, let $\omega_{x} \in\left[0, \omega_{2}\right]$, it can find $t$ when $\omega_{x}$ is at the minimum which means the optimization point is $\left(\omega_{\mathrm{x}}, \mathrm{a}_{\mathrm{x}}\right)$.

\section{Two - stage Exponential Acceleration Scheme and Iterative Algorithm Validation}

In order to verify the feasibility of the two-stage exponential acceleration scheme, the 57BYGH250C stepper motor and the Dvp-48EH model PLC are used as the main hardware to build the system. The parameters are shown in Table 1.

TABLE 1.System Parameters

\begin{tabular}{|c|c|c|c|}
\hline$\theta$ & $\mathrm{Tz}(\mathrm{N} \cdot \mathrm{m})$ & $\beta$ & $\mathrm{J}\left(\mathrm{Kg} \cdot \mathrm{m}^{2}\right)$ \\
\hline $0.9^{\circ}$ & 0.3 & 0.05 & 1.03 \\
\hline
\end{tabular}

And according to the data provided by the manufacturers, we draw the motor torque characteristics of the curve, based on system parameters, as shown in Figure 6, the critical load curve.
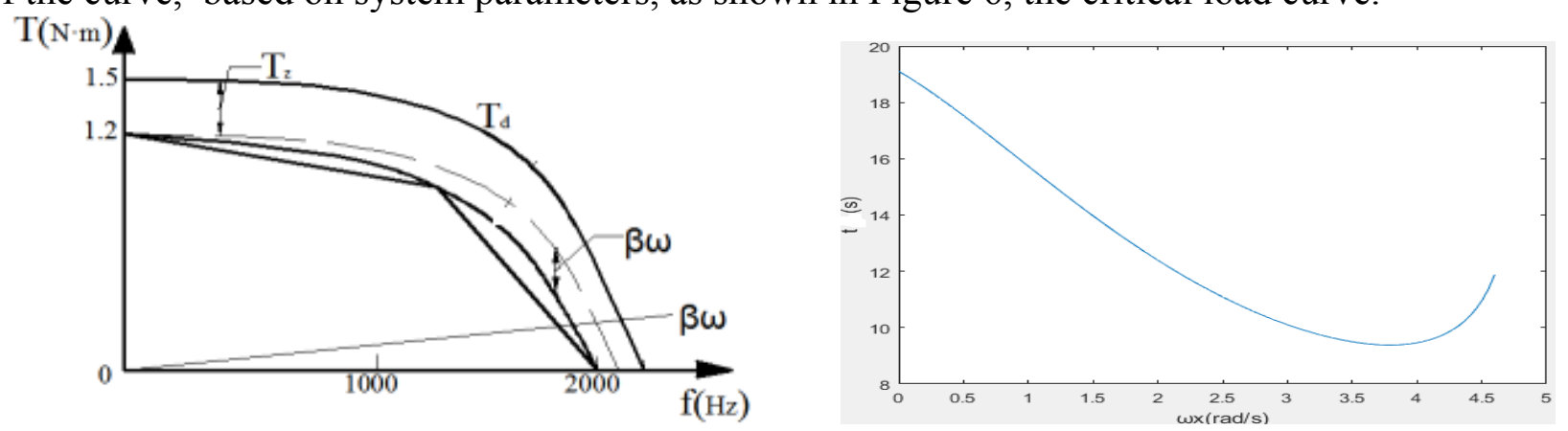

Figure 5. Two - Stage Exponential Acceleration Plan Figure 6. The Choice of the Best Break Point

Using Matlab to get Figure 7, we can find that the optimal point appear near $\omega=3.6 \mathrm{rad} / \mathrm{s}$, which shows that the acceleration process takes about $9 \mathrm{~s}$. a $2=1.2 \mathrm{rad} / \mathrm{s} 2, \omega 2=5 \mathrm{rad} / \mathrm{s}$, respectively, we used the exponential and two-stage Exponential acceleration algorithm.

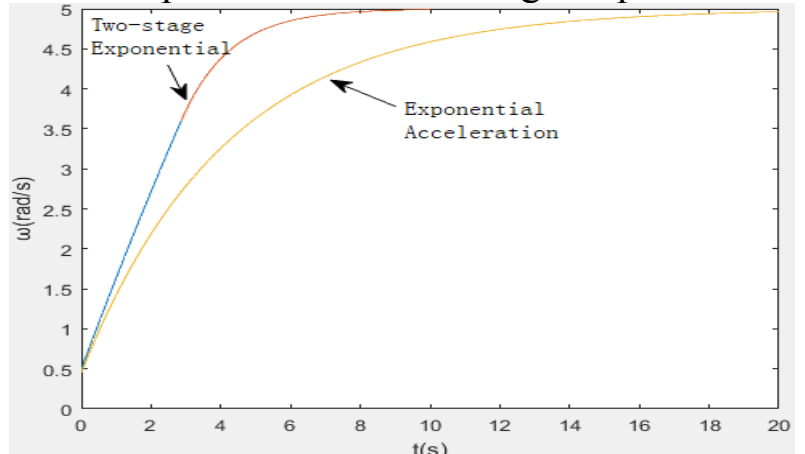

Figure 7. The Choice of the Best Break Point

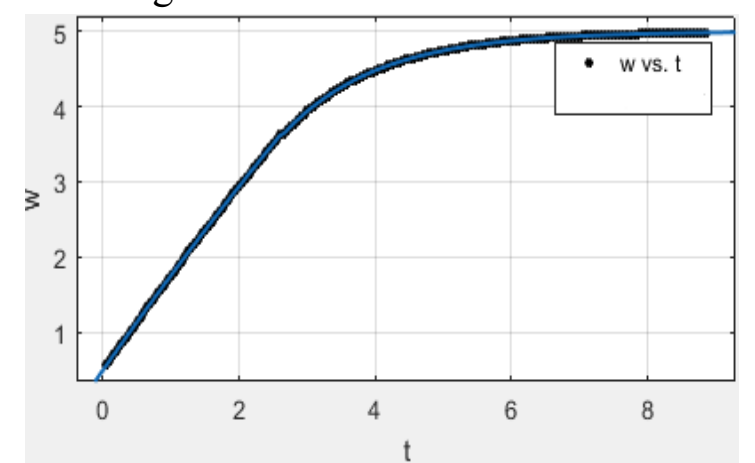

Figure 8. Speed Curve Fitting

Figure 7 shows the simulation results of the two algorithms. It can be found that the two-stage exponential linear acceleration algorithm is about half the speed of the exponential acceleration algorithm, about $5 \mathrm{rad} / \mathrm{s}$ at about $9 \mathrm{~s}$, and the exponential acceleration reaches the target speed of $5 \mathrm{rad}$ / s at $20 \mathrm{~s}$. 
Use VS2015 on the host computer programming, and based on the Modbus protocol [7], PC and PLC were establish serial communication between the line-line algorithm validation test. We the use of encoder to detect real-time motor speed, through the host computer program stored in the text file, after the introduction of Matlab curve fitting. Due to the accuracy of the encoder and the inherent delay of the system, the strategy of detecting the motor speed once every $50 \mathrm{~ms}$ is taken. Table 2 shows part of the encoder measured speed data.

TABLE 2. Two - Stage Exponentia Acceleration Plan

\begin{tabular}{|c|c|c|c|c|c|}
\hline $\mathrm{t}(\mathrm{ms})$ & $\omega(\mathrm{rad} / \mathrm{s})$ & $\mathrm{t}(\mathrm{ms})$ & $\omega(\mathrm{rad} / \mathrm{s})$ & $\mathrm{t}(\mathrm{ms})$ & $\omega(\mathrm{rad} / \mathrm{s})$ \\
\hline 50 & 0.56 & 5100 & 4.72 & 5600 & 4.80 \\
\hline 100 & 0.63 & 5200 & 4.73 & 5700 & 4.82 \\
\hline 150 & 0.71 & 5300 & 4.75 & 5800 & 4.83 \\
\hline 250 & 0.83 & 5500 & 4.79 & 5900 & 4.84 \\
\hline
\end{tabular}

After saving PC's speed of data import after the Matlab software, it now was shown in figure 8 speed fitting curve. It can be found that the speed curve and simulation curve is in the region of the conformity degree, proving the rationality of the secondary exponential iterative algorithm.

\section{Conclusion}

(1) Compared with the traditional exponential acceleration method, a two-stage exponential acceleration scheme based on the time-frequency curve is proposed. The scheme can make full use of the stepping motor output torque, and the acceleration effect is better than the exponential acceleration algorithm.

(2) Research on the selection of the inflection point of the second-stage exponential acceleration scheme. Using the method of fitting the critical load curve by Matlab can easily select the best point.

(3) By constructing the iterative equation algorithm to reduce the discrete degree of the acceleration curve, the experiment verifies the feasibility of the algorithm.

\section{References}

[1] Chen Yong-li, Li-li. Design of Robot Control System Based on PLC and Stepping Motor [J]. Journal of Anyang Teachers College, 2013, (2): 51-53.

[2] Chen-Ai-guo, Huang Wen-ling, Yang-Honghong. Research on the Speed - up Curve of Stepping Motor [J]. Development \& Innovation of Machinery \& Electrical Products, 2003, (2): 47-49.

[3] Zeng-Kanglin, Xiang-yu, Optimization and Realization of Exponential Acceleration Curve for Stepping Motor [J]. Micro-motor, 2014, 47(6): 36-40.

[4] Sun-Kong-zheng, Xu-Shuyan, Cao-Xiaotao, et al. Acceleration and Deceleration Control of Stepping Motor Based on Moment Frequency Characteristic [J]. Micro motors Servo Technique, 2014, 47(1): 81-85.

[5] Kang-Jing, Shao-Qiang, Hu-Hongying. Feedback control to improve the drive power frequency characteristics [J]. Small and Special Electrical Machines, 2008, (6): 34-36.

[6] Wang-Yong, Wang-Wei, Yang-Wentao. Design and Application of Step - down Curve Control System for Stepping Motor [J]. Control Engineering, 2008, 15(5): 576-579.

[7] Lu Wen-jun, Leng Shan, Yang Jian-jun. Controller Remote Monitoring System Based on Modbus Protocol [J]. Electric Power Automation Equipment, 2003, 23(06): 54-56. 